\title{
Rheumatoid arthritis and bone health
}

\author{
Authors: Shanze Ashai ${ }^{\mathrm{A}}$ and Nicholas $\mathrm{C}$ Harvey ${ }^{\mathrm{B}}$
}

Rheumatoid arthritis (RA) is an inflammatory arthropathy affecting $1 \%$ of the population, with a female predominance. Systemic inflammation is a key component of RA disease; corticosteroids are often required to rapidly control disease activity. Both inflammation and corticosteroids, however, have an adverse effect on bone mineral density, potentially resulting in osteoporosis and an increased risk of fractures. In this article, we describe the link between RA and impaired bone health, together with appropriate strategies to maintain bone density and reduce fracture risk. Key approaches include achieving adequate control of inflammation, minimising corticosteroid use, monitoring bone mineral density and intervening with antiosteoporosis medications when indicated.

\section{Introduction}

Rheumatoid Arthritis (RA) is a common chronic autoimmune inflammatory condition. It typically presents with a symmetrical polyarthritis associated with fatigue, pain and weakness. ${ }^{1}$ RA results in systemic inflammation and can also cause extraarticular manifestations. The disease affects about $1 \%$ of the British population, with the peak age of onset between 45 and 75 years. It is up to four times more common in women than in men. RA patients are at higher risk of fractures, falls and fall-related injuries compared with the healthy population. ${ }^{2}$ The National Institute for Health and Care Excellence (NICE) emphasises the need for early diagnosis and treatment to reduce the morbidity associated with RA; this review focuses on the risk of poor bone health in this group of patients.

\section{RA and bone}

The skeleton is constantly remodelled, adapting to the strains placed on it (eg as a result of physical activity and changing body mass) and to remove areas of microdamage. Thus, there is concomitant osteoclastic activity to resorb bone and osteoblastic activity to lay down a new osteoid matrix, which subsequently becomes mineralised.

RA causes bone loss that can be observed in three patterns: periarticular bone erosions, focal subchondral bone loss from

Authors: ${ }^{\text {A }}$ academic foundation trainee, MRC Lifecourse

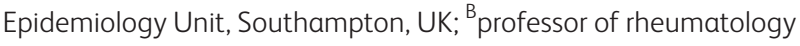
and clinical epidemiology, MRC Lifecourse Epidemiology Unit, Southampton, UK osteoclastic activity and systemic bone loss across the axial skeleton at least partly linked to systemic inflammation. ${ }^{3}$

Osteoporosis is a chronic disease characterised by the loss of bone mineral density (BMD) and increased fracture risk. It is more common in patients with inflammatory arthropathies. Osteoporosis can be found in $30-50 \%$ of patients with RA, depending on age and sex, with prevalence and fracture risk both increasing with disease duration and seropositivity. ${ }^{4}$ The risk of osteoporosis developing in either male or premenopausal female RA patients is double that of age and sex-matched healthy controls. ${ }^{5}$ RA disease activity has been shown to impact BMD: disease remission is associated with preservation of BMD, whereas even low to moderately active disease is associated with higher radiological RA damage and lower BMD at the hip. ${ }^{6}$ RA patients are twice as likely as healthy controls to experience hip fractures, which have considerable associated morbidity and mortality. ${ }^{7}$

\section{Key points}

Rheumatoid arthritis (RA) is an autoimmune inflammatory arthropathy of the synovial joints with multisystem involvement. It results in synovitis, bone destruction and systemic symptoms.

Systemic inflammation associated with RA, and corticosteroid treatment often used to manage the condition, lead to bone loss and increased risk of fractures.

Long-term minimisation of RA disease activity using non-corticosteroid medications (disease-modifying antirheumatic drugs and biologics) is the therapeutic target.

Bone health in these patients should be addressed with bone mineral density (BMD) assessment (dual-energy X-ray absorptiometry) and assessment of fracture risk $\left(\right.$ FRA ${ }^{\circledR}$ ).

Antiosteoporosis medication is likely to be indicated in older patients taking systemic corticosteroids for 3 months or longer, those with low BMD or those at high fracture risk using FRAX ${ }^{\circledR}$.

KEYWORDS: Rheumatoid arthritis, osteoporosis, epidemiology, fracture, glucocorticoid

DOI: 10.7861/clinmed.20.6.rabh 


\section{Management of bone health in RA: general measures}

RA is managed with a treat-to-target approach to achieve lowest possible disease activity. Drug therapy initiation is recommended within 12 weeks of presentation to achieve the best patient outcomes. $^{8}$

Pain may be relieved with non-steroidal anti-inflammatory drugs (NSAIDs) and steroids; while these medications may also help with early morning stiffness, they do not alter the underlying inflammation. Disease-modifying antirheumatic drugs (DMARDS), such as methotrexate, sulfasalazine and hydroxychloroquine, are therefore used to reduce inflammation and so control the disease process. Newer 'biologic' treatments, such as those targeting tumour necrosis factor-alpha and related inflammatory pathways are further, and increasingly used, options where traditional DMARDS offer inadequate disease control. DMARDS and biologics have not been convincingly associated with bone loss and increased fracture risk, over and above that due to the RA itself. ${ }^{9}$

In contrast, oral and parenteral corticosteroids have been clearly shown to rapidly increase fracture risk. The relationship appears continuous such that there is no safe lower dose of corticosteroid below which fracture risk is not increased. ${ }^{10}$ Corticosteroids impair osteoblastic activity and increase bone resorption by osteoclasts. Fracture risk is increased more than that expected from BMD loss, and declines swiftly after cessation of therapy.

Current guidelines recommend that antiosteoporosis therapy is considered in all patients anticipated to need three or more months of systemic corticosteroid therapy. ${ }^{11}$ In older patients, this may simply be started concomitantly with the corticosteroid; in younger patients (for example $<70$ years), a bone density scan (dual-energy $\mathrm{X}$-ray absorptiometry) may be recommended first. $R A$ is an input variable in the FRA $X^{\circledR}$ fracture risk calculator, which is used to calculate fracture probability over the next 10 years and can be linked with published treatment thresholds using the UK National Osteoporosis Guideline Group site..$^{11,12}$

\section{Antiosteoporosis medications}

Calcium and vitamin D supplementation appear to have very modest efficacy for reducing the risk of osteoporotic fractures when used together. They appear best directed at those expected or known to have inadequate intake/status of these nutrients and those taking antiosteoporosis medications rather than used as the sole therapeutic approach. ${ }^{13}$

\section{Bisphosphonates}

Bisphosphonates are synthetic analogues of the naturally occurring compound pyrophosphate and bind strongly to hydroxyapatite, inhibiting bone resorption by inactivating osteoclasts. The most frequently prescribed oral bisphosphonate is alendronate and is commonly the first-line treatment. If taken properly (in the morning with a glass of water, 45 minutes before food, drink or other medications and remaining upright for about 30-60 minutes after the dose), upper gastrointestinal side effects are uncommon. However, for those who are unable to tolerate oral bisphosphonates, or in whom they are contraindicated (for example because of malabsorption or dysphagia), then an intravenous bisphosphonate, such as zoledronate (given yearly in a dose of $5 \mathrm{mg}$ by infusion over a minimum of 15 minutes) is an alternative. $^{14}$

\section{Denosumab}

Denosumab, a fully humanised antibody to receptor activator of nuclear factor kappa B ligand (RANKL) is a newer antiresorptive agent. RANKL, secreted by osteoblasts, is a major activator of osteoclastic bone resorption. Denosumab mimics the action of osteoprotegerin (OPG), an endogenous inhibitor of this pathway. It is administered as a subcutaneous injection once every 6 months. Three-year fracture data show a $68 \%$ reduction in vertebral fracture and $40 \%$ reduction in hip fracture; efficacy and safety have been demonstrated to 10 years. Side effects are uncommon, but hypocalcaemia can occur, particularly if the patient is vitamin D deficient or has renal impairment. ${ }^{15}$ Rarely skin infections, predominantly cellulitis, may occur. ${ }^{14}$

\section{Selective oestrogen receptor modulators: raloxifene}

Raloxifene is a selective oestrogen receptor modulator (SERM) that has antiresorptive oestrogenic effects on the skeleton without the unwanted risks of oestrogen in the breast. Its use is also associated with a significant decrease in the risk of breast cancer. It has been shown to be effective in preventing post-menopausal bone loss and in preventing vertebral fractures. However, there is no evidence that raloxifene prevents hip or non-vertebral fractures. ${ }^{16}$ Adverse effects include leg oedema, cramps, hot flushes and a two to threefold increase in the risk of venous thromboembolism. ${ }^{14}$

\section{Teriparatide}

Teriparatide (recombinant human 1-34 parathyroid-hormone peptide) has truly anabolic effects on bone. ${ }^{14}$ It is administered by subcutaneous injection in daily doses of $20 \mu \mathrm{g}$. It increases bone formation and produces large increases in BMD, leading to approximately $70 \%$ reduction in the incidence of new moderate or severe vertebral fractures over 18 months of treatment, together with reductions in non-vertebral fractures. ${ }^{17}$ Side effects are uncommon but may include nausea, headache and dizziness; in addition, transient hypercalcaemia and hypercalciuria may occur. The use of teriparatide in the UK is currently limited by NICE guidance to those older patients at highest fracture risk and who may have failed other therapies.

\section{Adverse effects and duration of therapy}

Osteonecrosis of jaw is extremely rarely observed during therapy for osteoporosis ( $<1 / 100,000$ per year) for individuals on oral bisphosphonates, but appears more commonly when higher doses of bisphosphonates are given intravenously for treatment of bone metastases. ${ }^{18}$ A causal link to bisphosphonates is unproven but international guidance suggests a prudent approach, encouraging patients to maintain good oral hygiene and have regular dental visits, with invasive dental work performed before commencement of bisphosphonate or denosumab therapy. ${ }^{19}$ Atypical femoral fractures of the subtrochanteric region and femoral shaft may also rarely occur in patients taking bisphosphonates or denosumab. These are usually located in the lateral cortex around which endosteal thickening may be observed prior to fracture occurrence. Individuals may have prodromal pain and fractures typically are transverse, sometimes bilateral and occur after minimal trauma. These are rare events and the fractures prevented by treatment substantially outnumber any which might be a consequence of it. ${ }^{20}$ 
Current UK guidance suggests reassessment of the need for treatment after 3 years of intravenous bisphosphonate/ subcutaneous denosumab, and after 5 years of oral bisphosphonate. ${ }^{11}$ For high-risk patients, continuation of treatment is usually warranted, but where there have been no incident fractures and bone mineral density has improved, a period without treatment may be recommended. However, rapid bone loss and multiple vertebral fractures have been reported after cessation of denosumab, so this agent should be followed by a longer acting antiresorptive, such as zoledronate, if a treatment pause is decided upon. ${ }^{14}$

\section{Conclusion}

Osteoporosis is common in patients with RA and predisposes the patient to fractures which increase disability, mortality and morbidity. A treat-to-target strategy is most effective in delaying RA disease progression and appropriate management to control inflammation is important in reducing the risk of osteoporosis and fracture. Bone health is addressed through minimising inflammation using steroid sparing agents to ensure that the lowest possible dose of corticosteroids (ideally none) is used, assessing fracture risk and bone mineral density, and treating with antiosteoporosis medications if appropriate.

\section{References}

1 Ruddy S, Harris ED Jr, Sledge CB, Budd RC, Sergent JS (eds). Kelley's Textbook of Rheumatology, 7th edn. Philadelphia: Elsevier, 2005.

2 Stanmore E, Oldham J, Skelton D et al. Fall incidence and outcomes of falls in a prospective study of adults with rheumatoid arthritis. Arthritis Care Res (Hoboken) 2013;65:737-44.

3 Shimizu T, Takahata M, Kimura-Suda $\mathrm{H}$ et al. Autoimmune arthritis deteriorates bone quantity and quality of periarticular bone in a mouse model of rheumatoid arthritis. Osteoporos Int 2016;28: 709-18.

4 Hauser B, Riches P, Wilson J, Horne A, Ralston S. Prevalence, and clinical prediction of osteoporosis in a contemporary cohort of patients with rheumatoid arthritis. Rheumatology 2014;53:1759-66.

5 Haugeberg G, Uhlig T, Falch J, Halse J, Kvien T. Bone mineral density and frequency of osteoporosis in female patients with rheumatoid arthritis: Results from 394 patients in the Oslo County rheumatoid arthritis register. Arthritis Rheum 2000;43:522.

6 Lodder M. Bone mineral density in patients with rheumatoid arthritis: relation between disease severity and low bone mineral density. Ann Rheum Dis 2004;63:1576-80.

7 van Staa TP, Geusens P, Bijlsma JW, Leufkens HG, Cooper C. Clinical assessment of the long-term risk of fracture in patients with rheumatoid arthritis. Arthritis Rheum 2006;54:3104-12.
8 Aletaha D, Neogi T, Silman A et al. 2010 Rheumatoid arthritis classification criteria: An American College of Rheumatology/ European League Against Rheumatism collaborative initiative. Arthritis Rheum 2010;62:2569-81.

9 Clynes M, Jameson K, Prieto-Alhambra D et al. Impact of rheumatoid arthritis and its management on falls, fracture and bone mineral density in UK biobank. Front Endocrinol (Lausanne) 2019;10:817.

10 van Staa T, Leufkens H, Abenhaim L, Zhang B, Cooper C. Use of oral corticosteroids and risk of fractures. J Bone Miner Res 2000;15: 993-1000.

11 Compston J, Cooper A, Cooper C et al. UK clinical guideline for the prevention and treatment of osteoporosis. Arch Osteoporos 2017; 12:43.

12 Kanis J, Harvey N, Johansson H, Odén A, Leslie W, McCloskey E. FRAX Update. Journal of Clinical Densitometry 2017;20:360-7.

13 Harvey N, Biver E, Kaufman ] et al. The role of calcium supplementation in healthy musculoskeletal ageing. Osteoporos Int 2016:28:447-62.

14 Curtis E, Moon R, Dennison E, Harvey N, Cooper C. Recent advances in the pathogenesis and treatment of osteoporosis. Clin Med 2015;15(Suppl 6):s92-6.

15 Cummings S, Martin J, McClung M et al. Denosumab for prevention of fractures in postmenopausal women with osteoporosis. N Engl J Med 2009;361:756-65.

16 Ettinger B. Reduction of vertebral fracture risk in postmenopausa women with osteoporosis treated with raloxifene: results from a 3-year randomized clinical trial. JAMA 1999;282:637.

17 Neer R, Arnaud C, Zanchetta J et al. Effect of parathyroid hormone (1-34) on fractures and bone mineral density in postmenopausal women with osteoporosis. Obstetrical and Gynecological Survey 2001;56:623-4.

18 Khan A, Morrison A, Hanley D et al. Diagnosis and management of osteonecrosis of the jaw: a systematic review and international consensus. J Bone Miner Res 2014;30:3-23.

19 Khosla S, Burr D, Cauley J et al. Bisphosphonate-associated osteonecrosis of the jaw: report of a task force of the American Society for Bone and Mineral Research. J Bone Miner Res 2007;22:1479-91.

20 Shane E, Burr D, Abrahamsen B et al. Atypical subtrochanteric and diaphyseal femoral fractures: second report of a task force of the American Society for Bone and Mineral Research. J Bone Miner Res 2013:29:1-23.

Address for correspondence: Prof Nicholas Harvey, MRC Lifecourse Epidemiology Unit, University of Southampton, Southampton General Hospital, Tremona Road, Southampton SO16 6YD, UK.

Email: nch@mrc.soton.ac.uk

Twitter: @ProfNickHarvey 